\title{
Biochemistry Among the Stars
}

\author{
Physicist Mike Famiano explores the possibility that nuclear processes in \\ extreme astrophysical environments might be responsible for a puzzling \\ aspect of biochemistry.
}

\author{
By Marric Stephens
}

C

arl Sagan famously said that "we are made of star stuff." Few scientists understand the implications of that statement as well as Mike Famiano. A professor of nuclear astrophysics at Western Michigan University, Famiano's research focuses on stellar nucleosynthesis-the fusion of light elements into heavier ones within stars. Famiano also has a side project, one sparked by a passing conversation that he had as a graduate student roughly two decades ago. The goal of this project is to solve a problem related to one of the great mysteries of biology: life's overwhelming preference for biomolecules with a specific chirality, or "handedness." Famiano spoke to Physics about his interest in chiral molecules and his efforts to understand their origin.

\section{All interviews are edited for brevity and clarity.}

\section{What problem currently has your attention?}

Amino acids-the organic compounds that combine to form

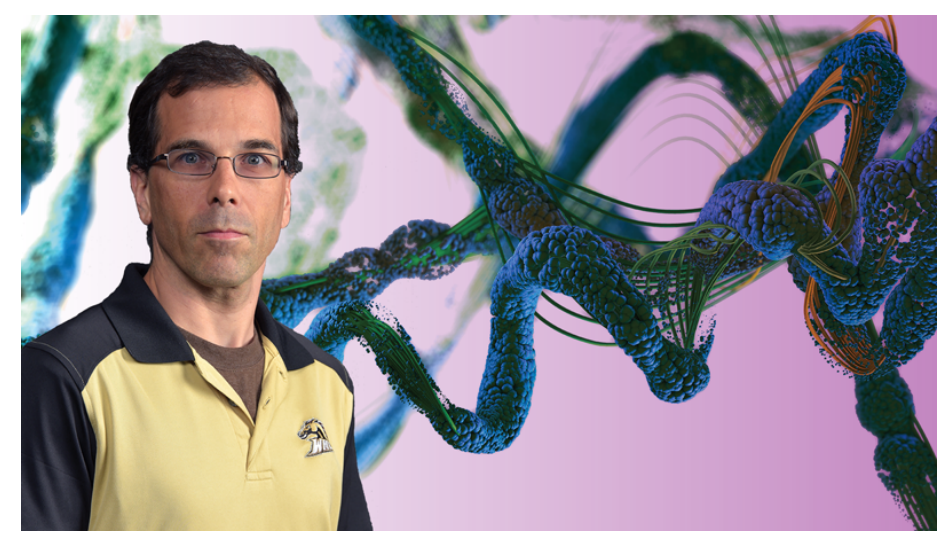

Credit: APS/Alan Stonebraker; Christoph Burgstedt/stock.adobe.com proteins-come in right- and left-handed forms. On Earth, all life uses the left-handed forms. Amino acids found in meteorites are also predominantly left-handed. These imbalances might be linked by a common cause, but we don't yet know what.

One prevailing theory is that life on Earth amplified the small imbalance seen in amino acids in meteorites. My colleagues and $I$ are running simulations to try to explain how that small imbalance in meteorites might have arisen.

\section{What mechanisms have been put forward to explain the imbalance?}

They fall broadly into two categories: stochastic processes and deterministic ones. Stochastic processes might involve some random energy fluctuation in the amino acid, for example. One such idea is that the first amino acid forms with a certain chirality, and then other molecules that happen to have the same chirality stack on top like bricks, excluding their opposite-chirality counterparts.

Deterministic processes are those that drive the direction of amino-acid chirality more directly. A popular deterministic mechanism involves circularly polarized light and has been experimentally demonstrated. That process has a lot of drawbacks, but our model does too.

\section{What is your model?}

The model involves asymmetries in the way certain particles interact with biomolecules at the nuclear level. It works through selective destruction: If there are initially equal numbers of left- and right-handed molecules and one kind is destroyed preferentially, then there's an imbalance.

My colleagues and I realized that a nucleus within an amino acid 
could be destroyed in a spin-polarized way via the weak force, a parity-violating interaction. To explore this idea, we looked first at destructive interactions involving spin-polarized leptons-specifically neutrinos, which are inherently chiral-and nitrogen atoms in amino acids. The destructive mechanism we explored is inverse beta decay. In that decay, the nitrogen atom becomes carbon-14 or oxygen-14, a process that destroys the whole amino acid. There's also a nuclear recoil, which can literally rip the molecule apart.

But this process is only chirality selective if the amino-acid molecules all align with each other. If that happens, only amino acids of one chirality will be pointing in a direction that allows their nitrogen atoms to interact with the neutrinos. This alignment could happen through crystallization, where the molecules all line up naturally, or it could happen in an electric field, for example.

\section{Are there any environments where the conditions required by your model come together?}

A neutron-star merger-with its high antineutrino flux-might have the right environment, although I'm a little skeptical about that scenario. Another possibility is a debris field orbiting an existing neutron star, which emits antineutrinos as it cools. Amino acids might form in the material produced in that system.

Another promising environment is that created by the supernova of a type of massive star called a Wolf-Rayet star.
These stars blow out their outer envelopes before they explode, creating a lot of debris that can coalesce into asteroids. That environment provides the chemistry needed to make amino acids.

\section{To what extent can your proposal be tested?}

The effect my colleagues and I are looking at involves the interaction of a nucleus with a molecule, which is really the principle behind nuclear magnetic resonance (NMR). We plan to run an NMR experiment this year that could verify some of our calculations at the molecular level.

We're also planning another experiment that's probably a lot further off. Our model should work for any polarized lepton, not just neutrinos. Facilities such as the Thomas Jefferson National Accelerator Facility produce polarized electrons-another lepton-so we could use those beams to test our idea. Natural scenarios involving neutrinos are easier to conjure because neutrinos are intrinsically chiral; however, environments containing spin-polarized electrons could also exist. Some of my colleagues don't really care to explore that option, but I want to explore everything. With all the possibilities to consider, I feel like a kid in an amusement park right now.

Marric Stephens is a Corresponding Editor for Physics based in Bristol, UK. 